\title{
Aspectos legales y documentales de las redes sociales: el modelo Facebook
}

\author{
Legal and documentary aspects of social web sites: the Facebook model
}

\section{Juan Miguel Sánchez Vigil (1), Juan Carlos Marcos Recio (2), Ricardo Villegas TovaR (3) y María Olivera ZaLdua (4)}

Biblioteconomía y Documentación, Adv. Ciudad Universitaria, S/N, Universidad Complutense, jmsvigil@telefonica.net, (2) jmarcos@ccinf.ucm.es, (3) Biblioteca "Niels Bohr”, Ciudad Universitaria, Benemérita Universidad Autónoma de Puebla, Puebla, México, ricardo.villegas@dgb.buap.mx, (4) Facultad de Ciencias de la Información, maryolizal@yahoo.es

\section{Resumen}

Estudio de los aspectos legales y documentales en las redes sociales, tomando como modelo la red Facebook. Se analiza la normativa legal en relación con el uso de los contenidos y las propuestas de Facebook. Se determina la tipología de los documentos en la red y se realiza el análisis global de los usos y aplicaciones. Se valoran los contenidos que aportan y manejan los usuarios, así como sus pretensiones y actitudes

Palabras clave: Redes sociales. Legislación sobre redes sociales. Documentación. Documentación en redes sociales. Facebook. Usuarios.

\section{Introducción}

Los datos que arroja Facebook por si solos son para llamar la atención: el número de suscriptores, el número de fotografías cargadas, el corto tiempo desde su creación, la cantidad de horas que dedican a la semana sus usuarios, etc. Todos ellos dan origen a una serie de análisis de muchos tipos. El que se expone aquí versa en su primera parte sobre los dos elementos más relevantes desde la perspectiva jurídica de esta red social: la privacidad y el derecho de autor. Ambas ramas han encontrado en Facebook una interrogante a sus propios principios, no solo por su aplicación, sino por el desconocimiento que los usuarios tienen del alcance de las políticas de uso. Para abundar en datos comparativos, en la segunda parte de este trabajo se muestran los resultados de una encuesta que busca ubicar los usos y razones que llevan a estudiantes universitarios a dedicar tiempo al uso del sistema. Para esto, se han encuestado a alumnos de diferentes facultades de la Universidad Complutense de Madrid y la Benemérita Universidad Autónoma de Puebla en México. Los datos que se muestran evidencian concordancias y diferencias que bien pu-

\begin{abstract}
The legal and documentary aspects of social networks are studied, using the Facebook network as the model. Legal regulations are analysed in relation with the use of contents and the proposals of Facebook. The type of documents found on the network is determined and an overall analysis is made of its uses and applications. The contents provided and handled by the users are evaluated, as well as their goals and approaches.
\end{abstract}

Keywords: Social networks. Legislation on social networks. Documentation. Documentation on social networks. Facebook. Users.

dieran dar origen a futuros trabajos sobre la temática.

$Y$ frente a Facebook, los medios tradicionales y también los digitales creen que las redes sociales han de complementar la información que ellos publican. En este sentido, las fuentes de información en los medios experimentan una profunda transformación. Así, algunos autores, entre ellos Chomsky cuestionan un asunto que los expertos dan por sentado (Celis, 2009):

\footnotetext{
Las fuentes de información todavía están en la prensa tradicional. Internet te da una variedad de opiniones, pero si realmente quieres saber los hechos, que es lo que esta pasando en los sitios, las opciones siguen siendo las mismas. No hay tantas fuentes de información como parece. Yo creo que la prensa tradicional va a sobrevivir. Encontrarán una manera de entender y utilizar la red en su propio beneficio. Eso sí, la calidad sigue desminuyendo, la información es hoy más homogénea que nunca.
}

Y frente a este planteamiento algo caduco están las redes sociales creciendo a un ritmo de miles de usuarios cada minuto. 


\section{De la normativa legal sobre redes sociales}

La primera década del siglo XXI no solo quedará registrada en la historia de las TICs por el desarrollo de dispositivos de almacenamiento y procesamiento de información de dimensiones diminutas y con enormes capacidades, sino que también estará marcada por la creación de interfases que por sus funciones han permitido que millones de personas interactúen como nunca antes se había visto. Existe una notoria tendencia a compartir archivos especialmente de vídeo, música y fotográficos, como lo indica la serie de reportes sobre el uso de Internet, a través de páginas electrónicas de redes sociales, sistemas P2P, blogs u otros de la tendencia Web 2.0

Todos estos cambios se dan en el contexto de la sociedad de la información, que se caracteriza por tener una incesante necesidad de obtener, manipular y transferir datos para una multitud de fines. Ante esta realidad las ciencias jurídicas no pueden quedarse al margen, ya que están en juego elementos esenciales de la convivencia social. Así pues la privacidad y los derechos de autor son las primeras áreas del derecho que han encontrado en sistemas tales como Facebook un reto a la aplicación de los principios que los regulan.

\subsection{Privacidad}

La tecnología que actualmente se encuentra disponible en las computadoras personales permite en breves momentos realizar actos que en el pasado consumirían importantes recursos económicos y de tiempo. Tomar fotografías, almacenarlas, editarlas, cargarlas en una red social y compartirlas es algo tan cotidiano que pocas veces se toma conciencia de ello. Sin embargo, con la ejecución de esos pasos bien podrían estarse afectando intereses de terceros que, dado el caso, serían imposibles de resarcir; más aún, uno mismo podría estar perdiendo de vista la línea que divide nuestra vida pública de la privada.

Es precisamente por la complicación que conlleva definir elementos tan subjetivos como la privacidad que el derecho no puede fácilmente enlistar un catálogo de prácticas definitivas y de aceptación general sobre qué imágenes o textos deben cargarse o no en las redes sociales. Tanto los tratados internacionales como las legislaciones nacionales se han ocupado de hacer respetar la esfera personalísima de los individuos, pero poco puede hacer el Estado cuando es el individuo el que por voluntad propia o ignorancia renuncia a esos derechos y abre la puer- ta para que cualquier persona pueda acceder a los perfiles que ella o él han elaborado. Este comportamiento ha dejado al descubierto datos como lugar y fecha de nacimiento, estado civil, información de contacto, creencias religiosas, posturas políticas, formación y ocupación, relaciones familiares y afectivas, permitiendo a quien las consulte ubicar el estilo de vida que lleva esa persona. Se han dado casos en los que la amplia visibilidad de estos datos ha dado origen a la ejecución de actos delictivos, especialmente por medio de estafas. En consecuencia, han surgido movimientos sociales como TRUSTe (http://www.truste.org/) que promueven una toma de conciencia sobre la importancia de resguardar la información personal. Los propios administradores de las redes sociales han tomado acciones: Facebook o MySpace prohíben su acceso a menores de 13 años y a los jóvenes entre 14 y 18 años les recomiendan suscribirse con el consentimiento de sus padres.

El rol que juega Facebook sobre el tema de la privacidad es crucial, ya que la esencia del sistema radica en compartir información de carácter personal y privada y, en consecuencia, establecer una red que por los perfiles de los participantes permita ubicar personas afines a ellos. Para esto, el sistema pondrá a disposición del usuario una serie de herramientas tecnológicas por él desarrolladas o por otras personas o entidades sobre las que no tiene control. Así, el usuario cargará tanta información personal como quiera, apoyándose en los propios delimitadores de privacidad que le permita la plataforma. Por tanto, es la ética de las personas la que decide qué es lo que ha de publicar y qué es lo que mantendrá en la secrecía.

\subsection{Debilidades de Facebook}

Una de las debilidades de Facebook es el manejo de la información que alberga en sus servidores. De acuerdo a su política de privacidad, guarda el tipo de navegador que está utilizando el usuario junto con su dirección IP (Facebook, 2008). Esta información no sólo le indica a la organización la ubicación geográfica del usuario, sino que en combinación con la información de su perfil, le permitiría crear una matriz de datos sobre los comportamientos del usuario. La empresa argumenta que esto lo realiza para fines de personalizar las características del sistema, pero además de eso, según la propia política, esta información puede ser compartida con empresas para desarrollar campañas publicitarias más exitosas. Para alcanzar este y otros objetivos Facebook puede recabar información del usuario no solo por lo que describe en su perfil, sino por lo que se publica acerca de él en 
periódicos o fuentes de Internet. Más aún, Facebook o sus empresas afiliadas pueden descargar en el ordenador del usuario cookies u otras tecnologías de análisis de comportamiento del usuario, tales como insignias de página Web, conocidas también como gifs $1 \times 1$. Los datos recabados por estás técnicas son suficientes para utilizar análisis como el data mining y ubicar áreas de oportunidad de negocio. Aunque Facebook declara que los datos de contacto de los usuarios no son compartidos con empresas, sí podría vender los resultados de sus análisis.

Facebook declara utilizar servidores protegidos con firewalls, pero se sabe que no existen sistemas capaces de asegurar la vigilancia de los datos y mucho menos cuando estos han sido cargados deliberadamente por el usuario. De ahí, la difícil aplicación de leyes en materia de protección de datos personales, pues poco puede hacerse desde los tribunales para controlar la diseminación de datos que el propio usuario ha puesto a disposición del público.

Como un medio de solución a posibles controversias sobre la privacidad, Facebook ha conformado un grupo de trabajo al que se le pueden exponer quejas por medio de un formato disponible en su plataforma. La solución que ofrezca este equipo no tendrá efectos legales y no podrá ir más allá del cierre de una cuenta de usuario o la notificación a una empresa afiliada a Facebook sobre la existencia de la queja, pero al menos prevalecerán los derechos del interesado para exponer su molestia ante un tribunal y corroborar si le asiste o no el derecho.

\subsection{Derechos de autor}

El derecho de autor, desde su nacimiento como rama jurídica, ha estado basado en una dualidad en cuanto a su espectro de acción. Por una parte, se encarga de la protección de las obras literarias o artísticas como expresiones humanas; y, por otra parte, promueve el acceso a ellas. Es decir, el ánimo de la ley es crear un equilibro entre el derecho a la compensación al autor por su trabajo y el derecho de acceso a la obra. Sin esta mediación no podría compartirse el conocimiento ni tampoco generarse nuevo. El conflicto no radica en reconocer la existencia del derecho de autor o el derecho de consulta de las obras, sino que ambos se contraponen al estar jerárquicamente en condiciones iguales. Ambos son derechos humanos y son reconocidos por una multitud de tratados internacionales, constituciones y demás legislaciones nacionales. Un antiguo principio del derecho hace alusión a que el fin de una facultad o privilegio está delimitado por el origen o principio de otro. Sin embargo, al igual que sucede al tratar de diferenciar lo público de lo privado, no existen reglas matemáticas o indicaciones de aplicación exacta que informen cuándo el uso de una obra literaria o artística sin el permiso del autor es legal o ilegal. Es cierto que existen excepciones y limitaciones que se aplican al derecho de autor para demarcar el alcance del monopolio que representa su aplicación, pero siempre es necesario hacer un análisis del caso concreto, no así realizar una interpretación generalizada.

La existencia de nuevas tecnologías ha exponenciado la problemática que representa la aplicación del derecho de autor y su contraposición con el derecho a la información, pues cada vez surgen nuevas aplicaciones que ponen en incertidumbre las indicaciones legales de una y otra rama jurídica. Desde la perspectiva del derecho de autor, las aplicaciones de la Web 2.0 también tienen su propia dualidad. Por una parte, son medios que permiten crear contenidos, inclusive de manera colectiva; y, por otra, ponen a disposición de los usuarios la capacidad de modificar o compartir contenidos que no son propios.

Para regular esta dualidad, Facebook ha dictado una serie de condiciones de uso que deben acatar todos sus usuarios sin posibilidad de negociar. De la interpretación de lo ahí vertido, es posible identificar tres grandes bloques en materia de derecho de autor que le aseguran a la organización un buen funcionamiento de su sistema:

1. Exigencia de originalidad de los contenidos cargados.

2. Cesión amplia de los derechos de autor sobre los contenidos a favor de los administradores de la plataforma.

3. Exclusión de responsabilidad de los administradores por los contenidos cargados en la plataforma.

Del primero se desprende que el titular de la cuenta se responsabiliza enteramente de los contenidos cargados, obligándose a no publicar, transmitir o compartir contenido que no sea de su propiedad o del que no tenga autorización para hacerlo. De no cumplirse con estas indicaciones, y a petición expresa del tercero afectado, los administradores de Facebook están en plena libertad de retirar los contenidos ilegales y de cerrar la cuenta del infractor. En caso de reincidencia, la solución prevista por los administradores es la expulsión definitiva de la red.

Han sido los propios administradores de Facebook quienes han desarrollado un formulario que permite denunciar presuntas violaciones a 
los derechos de autor por parte de personas que tengan cuenta dentro de la plataforma. En ella el afectado debe precisar cómo se ven vulnerados sus derechos y en qué parte se ubica dentro de la red social el archivo causante de la queja. Una vez que la solicitud haya sido revisada por los administradores, decidirán si eliminan el contenido informándole o no al responsable del acto. Ahora bien, éste último tiene la facultad de reclamar el borrado del archivo y le asistirá el derecho de revisión por los medios que la legislación norteamericana le otorgue. Si se llega al punto en que no se demuestra la violación, al denunciante se le puede acusar de perjurio e imputársele la sanción respectiva.

Este caso pone en relieve uno de los temas clave del derecho de autor y su aplicación en el contexto internacional. ¿Qué legislación debe aplicarse en caso de violación y qué tribunales son los que deben intervenir? En el contrato de condiciones de uso de Facebook se establece que el integrante de la red en caso de inconformidad deberá renunciar a la jurisdicción que le corresponda por su nacionalidad o residencia y someterse a la legislación aplicable al lugar donde opera la organización, es decir, al Estado de Delaware y a las leyes de aplicación nacional en los Estados Unidos de América.

El segundo aspecto da continuidad a la relación jurídica sobre contenidos que carga el usuario a la plataforma. Para que el material pueda ser legalmente colocado, es necesario que Facebook cuente con el permiso de almacenarlos y exhibirlos, de lo contrario la simple existencia del sistema encuadraría una violación a los derechos de los titulares por lo que en él se carga. La simple voluntad de parte del usuario de subir los contenidos a la plataforma no es justificante legal suficiente para que Facebook realice todos los actos que ejecuta. Por tanto, quienes cargan contenidos están obligados a ceder (Facebook, 2008a):

una licencia irrevocable, perpetua, no exclusiva, transferible, plenamente desembolsada y mundial (con derecho de sublicencia) para usar, copiar, reproducir públicamente, mostrar públicamente, reformatear, traducir, obtener extractos (totales o parciales) y distribuir dicho contenido de usuario para cualquier fin, ya sea comercial, publicitario o de otro tipo, en relación con el sitio o la promoción del mismo, para preparar trabajos, y para otorgar y autorizar sublicencias de lo anterior

A simple vista esta cláusula de uso puede identificarse como agresiva a cualquier titular de derechos de autor, ya que por medio de ella se está otorgando toda facultad sobre la obra a favor de la empresa para que ésta realice cualquier acto que le pueda convenir, incluyendo la de promocionar comercialmente los contenidos. Al ser expuesta esta cláusula el 23 de septiembre de 2008 en la página de Facebook, muchos usuarios mostraron su descontento e inclusive se inició una campaña para borrar perfiles y promover la no creación de nuevas cuentas. A pesar de la llamada de atención que esto representó para la empresa, ésta no ha modificado de manera sustancial el contenido de la cláusula, salvo en lo que respecta a la cesión, que ya no es de carácter exclusivo y el titular de los contenidos mantiene sus derechos. De remover lo cargado en el sistema, el usuario automáticamente revoca lo cedido, pero esto no obliga a Facebook a deshacerse de los respaldos que en su momento haya hecho.

Para el tercer aspecto (exclusión de responsabilidad de los administradores), se le exige al usuario que su actuación dentro de la red social no infrinja los derechos de terceros. En las condiciones de uso, Facebook va liberándose de responsabilidades, descargando éstas en el titular de la cuenta. Así por ejemplo, la exigencia de originalidad de lo cargado lo exime por contenido (Facebook, 2008a):

infractor, dañino, amenazante, ilegal, difamatorio, abusivo, incendiario, acosador, vulgar, obsceno, lascivo, fraudulento, que vulnere el derecho a la intimidad y la propia imagen o pueda exponer a la Compañía o a sus usuarios a cualquier tipo de daños o responsabilidades.

Además de la ventaja que representa para Facebook este planteamiento, existe otro más. El capítulo 17 sección 512 del Digital Millennium Copyight Act le exime de responsabilidad por cualquier daño o efecto que causen los contenidos del sistema al usuario de la plataforma o a otros.

\section{Uso de las redes sociales: modelo Facebook}

De las redes sociales se cuestionan fundamentalmente dos temas: la privacidad y el derecho de autor. En la primera, entran en juego decisiones personales; en la segunda, las leyes de propiedadad intelectual. En enero de 2009, Facebook (http://es-es.facebook.com/) contaba con cerca de diez mil millones de fotos y Flickr (http://www.flickr.com) con tres mil millones. Tal cantidad de documentos, a los que hay que añadir textos, videos, ilustraciones, etc., plantea la necesidad de un debate profundo desde las más altas instituciones internacionales.

En una aproximación general, con el fin de conocer algunos aspectos fundamentales sobre el uso de las mismas, elaboramos una encuesta distribuida entre una población universitaria (ver 
modelo anexo). Se realizaron 400 consultas (300 en España y 100 en México), en las Facultades de Ciencias de la Información y de la Documentación de la Universidad Complutense de Madrid, y en las Bibliotecas de Ciencias Naturales y Exactas e Ingenierías de la Benemérita Universidad Autónoma de Puebla de México. La media de edad del $80 \%$ de los estudiantes que participaron se sitúa entre los 18 y los 25 años; el $20 \%$ restante era mayor de 18 y menor de 40 años. El $98 \%$ de los encuestados utilizan alguna red social, y de éstos el $65 \%$ de los españoles y el $40 \%$ de los mexicanos pertenece a dos redes (bien Facebook y Tuenti http://www.tuenti.com/ en España, bien Facebook o MySpace http://www.myspace.com en México). El 15\% del total pertenece a tres o más redes.

\subsection{Relación con los demás}

Se adelanta una de las conclusiones básicas por resultar de interés para contextualizar: EI $75 \%$ de los usuarios no tiene otro interés que la relación con los demás o el intercambio de experiencias y en absoluto piensa en rentabilizar los contenidos; un $15 \%$ usa las redes para temas relacionados con el estudio o su formación; y un $8 \%$, significativo, entiende que le sirve para publicitarse y para buscar o bien mostrar su trabajo, tal y como se puede observar en la figura 1.
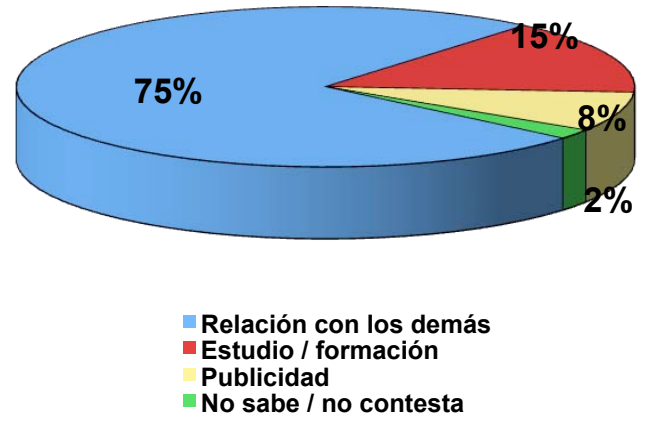

Figura 1. Datos sobre la relación con los demás en una red social

Se tomó como modelo Facebook por ser común en España y México, también más popular entre los encuestados. Al valorar las causas de alta en las redes, supimos que el $60 \%$ lo hizo por invitación de amigo, el $17 \%$ porque le gustaba, el $9 \%$ por moda, y un porcentaje en torno al $14 \%$ (60 de 400) declaró no tener una idea clara del porqué, pero que era consciente de que debía tener interés por el uso masivo. Solamente un $17 \%$ valoró los contenidos e hizo un análisis previo (elemental) de los contenidos de la página. Contrariamente a lo que se supone, el tiempo dedicado a las redes es mesurado: el $75 \%$ dedica menos de una hora diaria, el $13 \%$ entre 2 y 4 horas, y tan solo el $1 \%$ más de 4 horas.

Sobre los datos facilitados por el usuario, además de los nombres completos, direcciones, teléfonos, etc., la cuestión de la privacidad hace saltar las alarmas en preguntas sobre religión y política, cuestiones ciertamente "muy" privadas. El resultado indica que el $15 \%$ dio a conocer sus creencias religiosas y el $12 \%$ su ideología política. Sin embargo, no se aprovecha la facilidad de uso del correo electrónico mientras se consulta la página, ya que solo envían correos esporádicamente un $30 \%$, mientras que un $12 \%$ lo utiliza habitualmente

El principal uso de Facebook es para relacionarse con los amigos: el $80 \%$ no tiene otro interés, y destina la información solo a conocidos; mientras que el $25 \%$ la envía a toda la red de usuarios. Más de la mitad de los encuestados cuelga videos y fotografías, sin contemplar aspectos comerciales (apenas el $4 \%$ hacen referencia a este tema).

\subsection{Difusión de contenidos}

El material prioritario son las fotografías (52\%), más incluso que los textos del muro $(45 \%)$, y con diferencia de los vídeos (13\%), con una media de fotos colgadas por semana de entre 1 y 5 por usuario. La media de escritos es de uno diario, lo que significa que cada día circulan al menos tantas informaciones como número de usuarios. La casuística es ésta: el $70 \%$ envió entre 1 y 10 mensajes a la semana, el $15 \%$ entre 10 y 20 , el $4 \%$ entre 20 y 50 y el $3 \%$ más de 50 , como se observa en la figura 2.
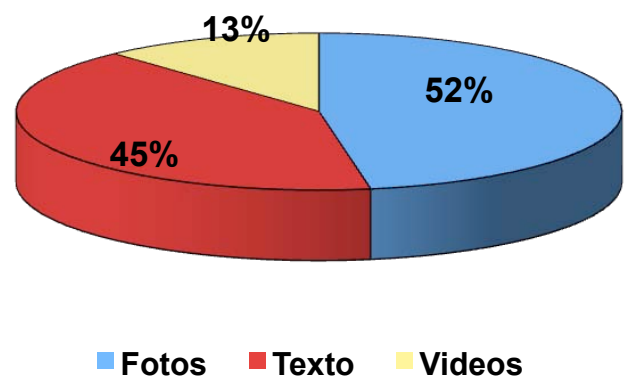

Figura 2. Reparto de fotos, texto y vídeos

\subsection{Contenido de las fotografías}

Los contenidos de las fotografías son generalmente personales (Figura 3). De familia y ami- 
gos, el $58 \%$; paisajes y retratos, el $20 \%$; y solo el $10 \%$ envió fotos de temas especiales o especializados. Los vídeos se reparten con un 35\% para la música, un $17 \%$ para escenas familiares o de la vida cotidiana, un $7 \%$ de viajes, otro $7 \%$ de cine, y, sorprendentemente, un $40 \%$ no da información (no contesta) sobre el tipo de vídeos que envía.

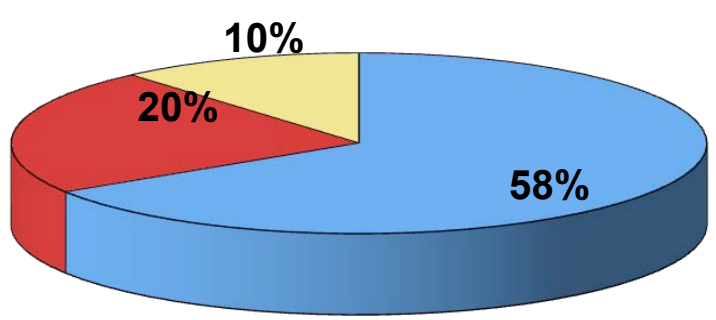

Familia y amigos

- Paisaje

Figura 3. Temas de las fotografías de Facebook

Una cuestión de interés fue saber por qué se cuelgan vídeos o fotografías de la vida privada y la respuesta fue por hobby en el $51 \%$ de los mexicanos y del $20 \%$ los españoles. Un $20 \%$ contestó que revelaba su intimidad para dar a conocer lo que hace, y solo un $4 \%$ confesó que el ego era lo que motivaba.

Un alto porcentaje, el $70 \%$ en España y el 55\% en México, no ha pensado en utilizar las redes para obtener beneficios, no ya económicos sino ni siquiera sociales o culturales. Para los mexicanos el mayor beneficio de la red (44\%) está en conseguir amigos, mientras que en España solo el $15 \%$ confiesa que es ese su propósito. Tampoco se contemplan las redes sociales como un elemento válido para encontrar trabajo o mejorar en el mismo (5\%)

\subsection{Derechos de autor}

En el terreno de los derechos de autor se produce una gran confusión, pues el $63 \%$ de los mexicanos y el $40 \%$ de los españoles no sabe que hay derechos sobre los contenidos. Una vez informados los encuestados sobre este aspecto, un $30 \%$ no consideró replantearse el uso o distribución de contenido; es decir, que le dio igual (Figura 4).

La posibilidad de comercializar los contenidos de los de las redes supuso también una sorpresa en las respuestas, pues el $75 \%$ de los españoles y el $54 \%$ de los mexicanos no autorizaría la venta de productos o contenidos ni siquiera negociando el precio. Entre el 20 y $30 \%$ autorizaría el uso si se abona una cuota.

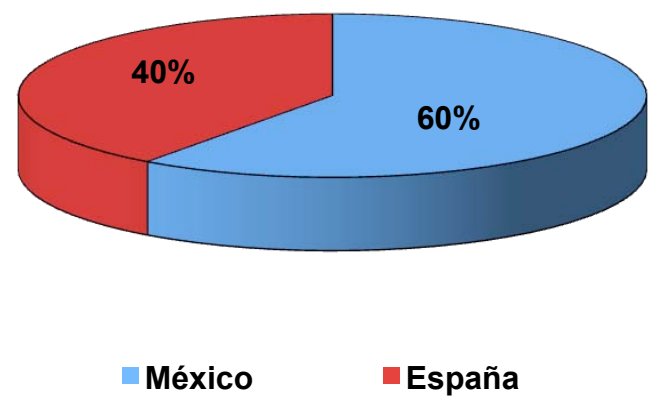

Figura 4. Los usuarios no conocen bien sus derechos

La cuestión de derechos resulta espinosa, ya que un $60 \%$ desconoce que el material que mueve de los demás y que reenvía, así como el que baja de la red, está sujeto a derechos. Sin embargo, la reacción fue positiva al informarles al respecto, puesto que el $75 \%$ en España y el $80 \%$ en México decidieron no facilitar información de otros al conocer que no eran los propietarios y que, por tanto, la decisión no era suya. Por otra parte, la frecuencia con que se mueve el material de los demás es del $15 \%$ en España y del $40 \%$ en México. Además, un $18 \%$ de los participantes continuaría facilitando información sujeta a derechos a pesar de saber que no podría o no debería hacerlo.

\section{Conclusiones}

1. Ha surgido una inquietud entre las empresas generadoras de contenidos por el mal uso que se ha hecho de las aplicaciones de sistemas basados en la tendencia Web 2.0, ya que han identificado que esto genera una reducción en sus ganancias debido a la transferencia ilegal de archivos de su propiedad.

2. Las empresas, por tanto, han emprendido una serie de acciones para aminorar estos efectos y reposicionar sus ventas, promocionando cambios en las leyes sobre derecho de autor para hacerlas más estrictas, presentando numerosas demandas, iniciando campañas de promoción al derecho de autor e, inclusive, implementando medidas tecnológicas de protección a sus contenidos.

3. En la práctica todos estos esfuerzos no son definitivos; solo reducen temporalmente la infracción a sus derechos. Lo cierto es que redes como las P2P siguen siendo las que más ocupan el tráfico en Internet y que los 
archivos que se están transfiriendo por ellas violentan los derechos de autor en un enorme porcentaje.

4. Contrariamente a lo que sucede con las redes P2P y la cuestionable legalidad de muchas de sus transferencias, en las redes sociales no sucede lo mismo, o al menos no en la misma magnitud. A pesar de que sí existe la posibilidad de compartir archivos por esos medios, no es una actividad recurrente el hacerlo sobre contenidos no propios. De los millones de fotografías cargadas en Facebook no se podría asegurar que un elevado porcentaje de ellas afectan los derechos de autor de terceros, dado que principalmente son creadas por los propios dueños de los perfiles y exhiben situaciones familiares o de amistad.

5. Facebook, el sistema ha sido diseñado con la finalidad de dar a conocer los perfiles de las personas a partir de comunicar por decisión propia información de carácter personal. Por otra parte, las cláusulas sobre el uso del servicio notifica al usuario, tanto al momento de la firma, como en repetidas ocasiones durante el uso del sistema, acerca de las obligaciones que tiene como tal usuario. En el caso de que se incurra en una infracción a los derechos de autor, ha previsto un mecanismo para resarcir el daño y en ausencia de efectividad de ese mecanismo, el sistema provee suficiente cantidad de información personal o de contacto del presunto responsable para entablar una demanda judicial y proceder conforme a derecho.

6. En cuanto a privacidad, Facebook no es el mejor aliado para mantener el anonimato de los suscriptores, pues a pesar de que existe una política definida sobre los comportamientos generalmente aceptados, es el propio usuario el que decide en qué medida da a conocer o no su perfil.

7. En un reciente estudio de Harris Interactive sobre las últimas estadísticas de uso de las redes sociales en EE.UU, se ofrece como resultado que casi la mitad de los estadounidenses participa en las redes sociales, un fenómeno que ha dejado atrás el calificativo de revolución silenciosa y ha entrado en muchos hogares del mundo a través de elementos participativos, bien sean fotografías, textos, viajes, aventuras, juegos, etc. Las redes sociales se hicieron mayores antes de que en algunos países se supiera que existían. Su fuerza es imparable y lo demuestra el número de altas en las mismas cada segundo, ya que se cuentan por miles.
8. En ese estudio se refleja que tres cuartas partes de las personas cuya edad figura entre 18-34 años (74\%) tienen una cuenta de MySpace o Facebook y sólo una cuarta parte $(24 \%)$ de los que tienen 55 años y de más edad tiene una cuenta. Tweeting también es ligeramente más frecuente entre los jóvenes, pero no por mucho: el $8 \%$ de $18-34$ años de edad usa Twitter, el $7 \%$ de los 35-44 lo utilizan; el $4 \%$ de los que tienen entre 45-54 años de edad; y sólo $1 \%$ de los que tiene 55 y más.

9. Estos datos reflejan que la gente joven es la que mejor ha sintonizado en las redes sociales, buscando una nueva forma de comunicar, de intercambiar datos y experiencias y de formalizar nuevas actitudes frente a la sociedad, alejadas de los medios tradicionales como han sido la televisión y en menor medida el resto de medios. También en España, como se demuestra en los datos aportados por esta investigación, el resto de medios está cediendo frente a Internet y el consumo de redes sociales. Una nueva generación despunta hacia la convivencia social, un terreno abonado para el crecimiento de experiencias entre los jóvenes sin muchas pretensiones globales pero con un sentimiento de compartir valores y propuestas interesantes para la comunidad a la que se dirigen.

\section{Referencias}

Arregocés Carrere, Benyi (2009). Manejar la privacidad en Facebook. // Consumer Eroski. http://www.consumer.es/ web/es/tecnologia/internet/2009/03/09/183489.php (13/03/2009).

Celis, Bárbara (2009). Noam Chomsky: No soy un don Quijote que mis molinos de viento son reales. // El País. Madrid. 2 de marzo.

Facebook. Condiciones de uso. http://www.facebook.com/ terms.php?locale=en_US (29/04/09).

Facebook. Política de privacidad. http://www.facebook. com/policy.php (29/04/09).

Facebook quiere convertirse en soporte para investigaciones comerciales. http://www.baquia.com/actualidad/ noticias/14561/facebook-quiere-convertirse-en-soportepara-investigaciones-comerciales (13-3-2009).

¿Quién es el dueño de la información en Facebook?. // Cadena ser: revista Web. http://www.cadenaser.com/ tecnologia/articulo/quien-dueno-informacion-facebook/c srcsrpor/20090217csrcsrtec_3/Tes (12-3-2009)

La Agencia de Protección de Datos pide a Facebook más garantías de privacidad. // La Razón. Madrid. 27 de marzo de 2009.

Facebook cede parte del control a los usuarios. // El País. Madrid. 22 de febrero de 2009.

Facebook no realizará cambios en materia de privacidad. // El Mundo. Madrid. 19 de febrero de 2009.

Ferrer-Sapena, Antonia y Javier Guallar (2008). Sobre la sociedad red. // El Profesional de la Información. 17-6 (2008) 689-692. 
Freire, Juan (2008). Redes sociales: ¿modelos organizativos o servicios digitales?. // El Profesional de la Información. $17: 6$ (2008) 585-588.

García, Iñigo (2009). Del Facebook a la Biblioteca Nacional. // El País. Madrid. 27 de febrero.

Grau, Abel (2008). ¿Sabe Facebook demasiado sobre sus usuarios?. // El País. Madrid. 1 de noviembre.

Jiménez Cano, Rosa (2009). La Biblioteca Nacional abre sus puertas a Facebook. // El País. 25 de febrero.

Jiménez de Luis, Ángel (2008). Las redes sociales están que arden, pero no de fiebres. En Ariadna. 368 (23 de marzo).

Krichel, Thomas (2008) Networks in digital libraries, a personal view. // El Profesional de la Información. 17:6 (2008) 662-663.

Just Under Half of Americans Have a Facebook or MySpace Account. The Harris Poll. http://www.harrisinteractive. com/harris_poll/pubs/Harris_Poll_2009_04_16.pdf (12-32009)

Leydesdorff, Loet; Thomas Schank, Andrea Scharnsorst y Wouter de Nooy (2008). Animating the development of social networks over time using a dynamic of multidimensional scaling. // El Profesional de la Información. 17:6 (2008) 611-627.

Margaix-Arnal, Dídac (2008). Las bibliotecas universitarias y Facebook: cómo y porqué estar presentes. // El Profesional de la Información. 17:6 (2008) 589-602.

Martín, Javier (2009). Internet no nos cambia tanto. // El País. Madrid. 26 de marzo.

McGirt, Ellen (2007). Haciendo amigos y millones. // Magazine. 430 (23 de diciembre).
Ortega, José Luis y Aguillo, Isidro F. (2008). Análisis estructural de una red social en línea: la red española de Flickr. // El Profesional de la Información. 17:6 (2008) 603-609.

Perianes-Rodríguez, Antonio; Carlos Olmeda Gómez y Féliz de Moya-Anegón (2008). Introducción al análisis de redes. // El Profesional de la Información. 17:6 (2008) 664669.

Peset, Fernando; Antonia Ferrer-Sapena y Tomàs Baiget 2008). Evolución social y networking en la comunidad biblio-documental. // El Profesional de la Información. $17: 6$ (2008) 627-636.

Rallo Lombarte, Artemi (2009). Los riesgos de Internet y las redes sociales. // Tribuna Complutense. (14 de abril) 4.

Riveiro, Aitor (2009). La democracia llega a Facebook. // El País. Madrid. 26 de febrero.

Los usuarios de Tuenti se declaran en huelga contra los términos de uso. // El País. Madrid. 3 de mayo.

Romero, Pablo (2008). El insaciable apetito de las redes sociales. // El Mundo. Madrid. 18 de enero.

Serrano, Irene (2009). Facebook, nueva herramienta para escritores y editoriales. // El Mundo. Madrid. 2 de marzo.

Torres Molina, Daniel (2008). Mark Zuckerberg, fundador de Facebook, en la Universidad de Navarra. // El Profesional de la Información. 1:-6 (2008) 681-684.

Uribe-Tirado, Alejandro; Andrés Felipe Echavarría-Ramírez (2008). Facebook como red de profesionales de bibliotecología, documentación y archivística en Iberoamérica. // El Profesional de la Información. 17:6 (2008) 670678.

Vander Veer, E. A. (2009). Exprimer Facebook. Madrid: Anaya.

\section{Anexo I: Redes sociales: facebook. Encuesta}

(Subraye la respuesta que proceda. Comente lo que considere al dorso de la segunda página.)

\section{Estudios}

Bachiller Universitario Licenciado Doctor Otros

Sexo H M

Trabaja SI No

Profesión

Estudiante Docente Oficio Administrativo

Profesión liberal Comercio Cultura Jubilado

Parado Otros

Edad

$\begin{array}{lll}\text { Menor } 18 & 18-25 & 25-40 \\ +40 & +50 & +60\end{array}$

Sí pertenece a una o varias redes sociales indique a cuales

Facebook Tuenti MySpace Otros

1. ¿Por qué se dio de alta en Facebook?

Invitación de amigo Está de moda Me gusta más que otros

Se lo dijeron Porque están todos

2. ¿Cuánto tiempo diario dedica a Facebook?

Menos de una hora $1-2$ horas

2- 4 horas Más de 4 horas

3. ¿Respondió a la pregunta sobre creencias religiosas?

Sí No No contesta
4. ¿Respondió a la pregunta sobre ideología política?

Sí No No contesta

5. ¿Envía los correos a sus amigos por Facebook en vez del E-mail personal?

Sí No A veces

6. ¿Usa Facebook para otras cuestiones que no sea relacionarse con amigos?

Sí No

7. ¿Para qué cuestiones?

Dar a conocer sus creaciones (fotos, videos, escritos)

Comercializar o vender algún producto

Hobby

Preguntar sobre temas de su interés

8. ¿Lo que cuelga en el muro lo da a conocer sólo a sus amigos o a todo Facebook?

Amigos Todos

9. ¿Qué material sube?

Fotos Videos Escritos en el muro

10. ¿Cuántas fotos a la semana?

Entre 1 y 5 Entre 5 y 10 Entre 10 y 20 + de 20

11. ¿Cuántos mensajes en el muro la semana?

Entre 1 y 5 Entre 5 y 10 Entre 10 y 20 + de 20

12. ¿Cuántas fotos ha colgado en el primer trimestre de 2009?

Menos de 10 Entre 10 y 20 Entre 20 y 50 + de 50 
13. ¿Cuántos videos ha colgado en primer trimestre de 2009 ?

Menos de 10 Entre 10 y 20 Entre 20 y 50 + de 50

14. ¿Cuántos escritos ha colgado en primer trimestre de 2009?

Menos de 10 Entre 10 y 20 Entre 20 y 50 + de 50 15. ¿Qué tipo de fotos?

Retratos personales Retratos de otros (amigos y familiares)

Vistas (paisaje de todo tipo) Fotografías especializadas

16. ¿Qué tipo de videos?

Personales y familiares Viaje Música Cines

17. ¿Por qué cuelga las fotografías y videos?

Dar a conocer lo que hace Ego personal (presumir)

Hobby Otras razones

18. ¿Piensa qué puede obtener algún beneficio?

Sí No

Buscar trabajo

Conseguir dinero

Hacer amigos
19. ¿Sabía que todo lo que cuelga está sujeto a derechos de autor?

$$
\text { Sí No }
$$

20. ¿Le da igual que esté sujeto a derechos?

$$
\text { Sí No }
$$

21. ¿Autorizaría a Facebook a que comercializara los contenidos que cuelga?

Sí, cobrando Sí, sin cobrar No

22. ¿Sabe que la documentación de otros en Facebook puede estar sujeta a derechos?

Sí No

23. Ahora que conoce el tema de derechos ¿Dará información sin analizarla antes?

$$
\text { Sí No }
$$

24. ¿Copia y pega con frecuencia los documentos de otros (fotos, videos, texto, etc.)?

$$
\text { Sí No }
$$

25. ¿Utiliza los contenidos de Facebook?

Si No

Trabajo Estudios Análisis Publicidad Empresas 